International Journal of Wireless \& Mobile Networks (IJWMN) Vol. 7, No. 4, August 2015

\title{
SINR, RSRP, RSSI AND RSRQ MEASUREMENTS IN LONG TERM EVOLUTION NETWORKS
}

\author{
${ }^{1}$ Farhana Afroz, ${ }^{1}$ Ramprasad Subramanian, ${ }^{1}$ Roshanak Heidary, ${ }^{1}$ Kumbesan \\ Sandrasegaran and ${ }^{2}$ Solaiman Ahmed \\ ${ }^{1}$ Faculty of Engineering and Information Technology, \\ University of Technology, Sydney, Australia \\ ${ }^{2}$ Department of Electrical and Electronic Engineering, \\ University of Dhaka, Bangladesh
}

\begin{abstract}
The four basic Radio Resource Management (RRM) measurements in Long Term Evolution (LTE) system are Channel Quality Indicator (CQI), Reference Signal Received Power (RSRP), Reference Signal Received Quality (RSRQ), and Carrier Received Signal Strength Indicator (RSSI). A measurement of channel quality represented by Signal to Interference plus Noise Ratio (SINR) is used for link adaptation along with packet scheduling, whereas RSRP and RSRQ are needed for making handover decision during intra-eUTRAN (evolved Universal Terrestrial Random Access Network) handover in LTE. In this paper, some practical measurement results recorded from a live LTE network of Australia using a commercial measurement tool namely NEMO Handy are analysed to verify the possible relationships among SINR, RSRP, RSSI and RSRQ as well as to evaluate the effects of SNR on throughput. In addition, the intraeUTRAN handover events occurred during the test period within the test area are studied. The analysis yields some useful information such as: if the SINR is good for a measurement slot, higher throughput is achieved; RSRP and SNR are proportional to each other on average; and lesser is the difference between RSSI and RSRP, better is the RSRQ - each of which is consistent with theory. All the measurement results are evaluated using computer programs built on MATLAB platform.
\end{abstract}

\section{KEYWORDS}

RRM, SINR, RSRP, RSSI, RSRQ, Link Adaptation, Packet Scheduling, Throughput, Handover

\section{INTRODUCTION}

In recent years, the demand of accessing high data rate applications on mobile device has noticeably increased. LTE, commonly marketed as 4G, is the 3GPP-standardized OFDMA/SCFDMA (Orthogonal Frequency Division Multiple Access in downlink/ Single Carrier Frequency Division Multiple Access in uplink) based wireless cellular system, which mainly focuses on providing high peak data rates, high spectral efficiency, improved capacity and coverage, short round trip time, and spectrum flexibility $[1,2,3]$. LTE radio access network, usually termed as eUTRAN, consists of eNodeBs only where all RRM-related functions are performed. RRM ensures that the air interface resources are efficiently utilized so that network efficiency is maximised, Quality of Service (QoS) required for each service/bearer/user be guaranteed, and high capacity and planned coverage are maintained. The RRM functionalities comprise of a range of algorithms employed for the optimal utilisation of radio resources. LTE RRM functions can be categorized as network based functions and connection based functions [4]. The network based DOI : 10.5121/ijwmn.2015.7409 
RRM functions, which are performed for a whole cell, include packet scheduling, admission control and load control, whereas the connection based functions such as power control and handover control are executed per connection basis. Among these RRM functions, packet scheduling is the process by which available radio resources are allocated among active users in order to satisfy their QoS requirements [5]. The smallest unit of radio resource that can be allocated to a user for data transmission is called Physical Resource Blocks (PRBs). The main objectives of packet scheduling are to maximize the cell capacity, to satisfy the minimum QoS needed for connections, and to maintain adequate resources for best-effort users with no strict QoS requirements [6]. In channel dependent scheduling, scheduling decisions are made based on channel conditions. The basic principle of channel dependent scheduling is to allocate PRBs to the users with relatively better channel conditions, and to avoid PRB allocation to the users experiencing deep fades. All users need to report the CQI computed by decoding the reference signals to the eNodeB which selects the user for data transmission and determines MCS (Modulation and Coding Scheme) based on instantaneous channel quality. CQI is a quantized and scaled version of the experienced SINR. The process of adapting MCS depending on current channel conditions is termed as Link Adaptation. If the SINR is good, higher order MCS (e.g. 64QAM) can be selected implying that more bits per modulation symbol can be transmitted and higher throughput can be achieved. If the SINR is poor, lower order MCS (i.e. QPSK) should be selected implying fewer bits per symbol are transmitted which in turn results in lower throughput. Other important RRM function is making handover (HO) decision based on handover algorithms. LTE supports break-before-make HO or hard HO only. In general, LTE HO process is divided into three steps such as measurement, judgement, and execution among which measurement is a crucial step that can directly affect HO performance [7]. Two measurement criteria such as RSRP and RSRQ are used to make a cell reselection or HO decision. When the RSRP and/or RSRQ of the serving cell fall(s) below the RSRP and/or RSRQ of the neighbor cell by a predefined HO margin for certain period of time, handover occurs. Fig. 1 shows that when a RSRP of a neighbor cell exceeds the signal strength of a serving cell by a predefined HO margin for certain duration, handover triggers. This paper provides an experimental study on the LTE measurements such as SINR, RSRP, RSSI and RSRQ to find the possible relationships among them as well as to analyse the effects of SNR on throughput. Furthermore, the intra-eUTRAN handover events occurred during the test period within the test area are studied. Some practical measurement results recorded from a live LTE network of Australia using a commercial measurement tool namely NEMO Handy are analysed for these purposes.

The rest of this paper is organized as follows. Section 2 illustrates an overview of each of these four LTE measurements followed by the obtained results and discussion in Section 3. Finally, Section 4 concludes the paper.

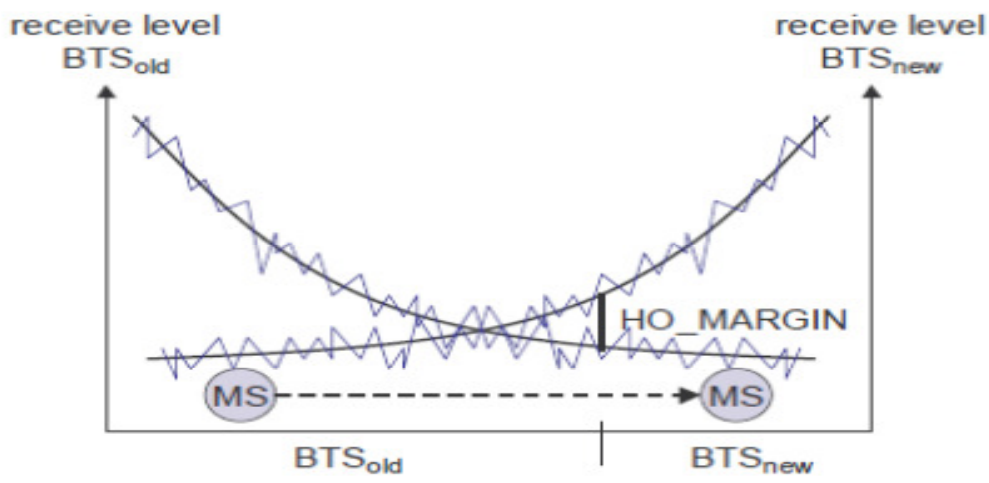

Figure 1: A typical handover scenario [8] 
International Journal of Wireless \& Mobile Networks (IJWMN) Vol. 7, No. 4, August 2015

\section{OVERVIEW OF LTE MEASUREMENTS}

In this section, the UE-related measurements in LTE such as SINR, RSRP, RSSI and RSRQ are illustrated as follows.

Signal to Interference plus Noise Ratio (SINR) is measured by UE on Resource Block (RB) basis. UE computes SINR on each RB, converts it to CQI and reports it to eNodeB where it is used to select the most suitable MCS for user data transmission in particular RB. SINR value defines the MCS to be used for a RB i.e. the number of bits per modulation symbol to be sent i.e. throughput to be achieved for that particular RB as well as the number of RBs to be allocated by eNodeB to user [9]. SINR can be defined as the ratio of the signal power to the summation of the average interference power from the other cells and the background noise.

Reference Signal Received Power (RSRP) is a cell-specific signal strength related metric that is used as an input for cell resection and handover decisions. For a particular cell, RSRP is defined as the average power (in Watts) of the Resource Elements (REs) that carry cell-specific Reference Signals (RSs) within the considered bandwidth [10].

RSRP measurement, normally expressed in $\mathrm{dBm}$, is utilized mainly to make ranking among different candidate cells in accordance with their signal strength. Generally, the reference signals on the first antenna port are used to determine RSRP, however, the reference signals sent on the second port can also be used in addition to the RSs on the first port if UE can detect that they are being transmitted $[10,11]$.

Reference Signal Received Quality (RSRQ) measurement is a cell-specific signal quality metric. Similar to the RSRP measurement, this metric is used mainly to provide ranking among different candidate cells in accordance with their signal quality. This metric can be employed as an input in making cell reselection and handover decisions in scenarios (for example) in which the RSRP measurements are not sufficient to make reliable cell-reselection/handover decisions. It is defined as [10]:

$R S R Q=(N \cdot R S R P) /(L T E$ carrier $R S S I)$

where, $\mathrm{N}$ is the number of Resource Blocks (RBs) of the LTE carrier Received Signal Strength Indicator (RSSI) measurement bandwidth.

Received Signal Strength Indicator (RSSI) is the linear average of the total received power observed only in OFDM symbols carrying reference symbols by UE from all sources, including co-channel non-serving and serving cells, adjacent channel interference and thermal noise, within the measurement bandwidth over N RBs $[10,11]$. RSSI is used as an input to compute the LTE RSRQ measurement discussed above.

From the above Eq. (1), it is seen that due to the inclusion of RSSI, RSRQ considers the combined effect of signal strength and interference. It can also be observed that mathematically RSRQ is proportional to RSRP.

RSRP, RSRQ and RSSI measurements are defined by 3GPP, however SINR is not defined in 3GPP specifications; it is specified by UE vendors. 


\section{RESULTS AND DISCUSSION}

In this section, some practical measurement results recorded from a live LTE network of Australia using a commercial measurement tool namely NEMO Handy are analysed to verify the possible relationships among LTE measurement parameters such as SINR, RSRP, RSSI and RSRQ as well as to evaluate the effects of SNR on throughput (Case 1). Furthermore, the intraeUTRAN handover events occurred during the test period within the test area are studied (Case 2). All the measurements results are analysed using computer programs built on MATLAB platform. The mobility scenarios along with the discussion of the obtained results are reported in following subsections.

\subsection{Case 1: Study of LTE Measurements}

In Case 1, a mobility scenario with the NEMO handset moving at a pedestrian speed from an area with good signal quality to the cell boundary where there is no adjacent cell to make HO to (i.e. death spot), is considered. The measurement results were recorded while the handset was downloading a file. The considered LTE network was an LTE FDD network with a bandwidth of $10 \mathrm{MHz}$ that corresponds to 50 PRBs. The measurement was taken over 188 measurement slots. Unlike 'scheduling slot', the term 'measurement slot' refers to the time scale when the NEMO handset could record the data.

\subsubsection{Relationship between SNR and RSRP}

In this subsection, the SNR and RSRP graphs are compared to observe the possible relationship between them. Comparing the SNR and RSRP graphs in Fig. 2 and Fig. 3 respectively, it can be observed that as a whole both SNR and RSRP show a decreasing trend with increasing measurement slots. As mentioned earlier the measurements were conducted while the handset was moving from an area with good signal quality to the cell boundary where there is no adjacent cell to handover. As a consequence, theoretically the SNR should be higher at the starting measurement slot and progressively should be lower as the handset is moving toward cell edge. In addition to this, if SNR is higher, channel quality is better i.e. RSRP will be better. It can be noticed from the figures that the RSRP and SNR graphs follow this trend on average implying that RSRP and SNR are proportional to each other on average.

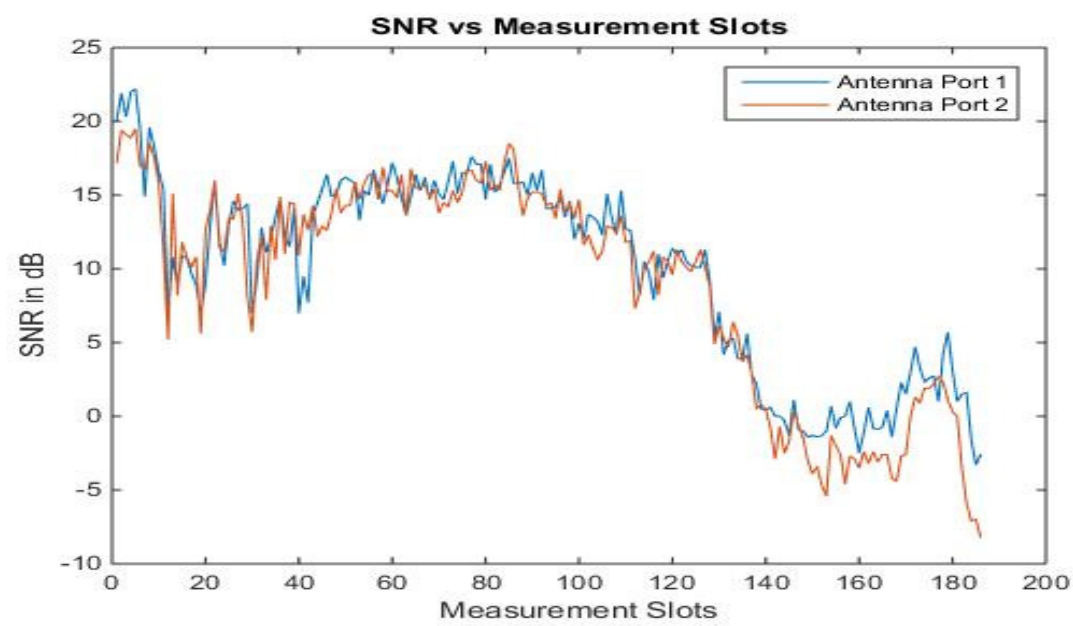

Figure 2: RS SNR versus measurement slots 


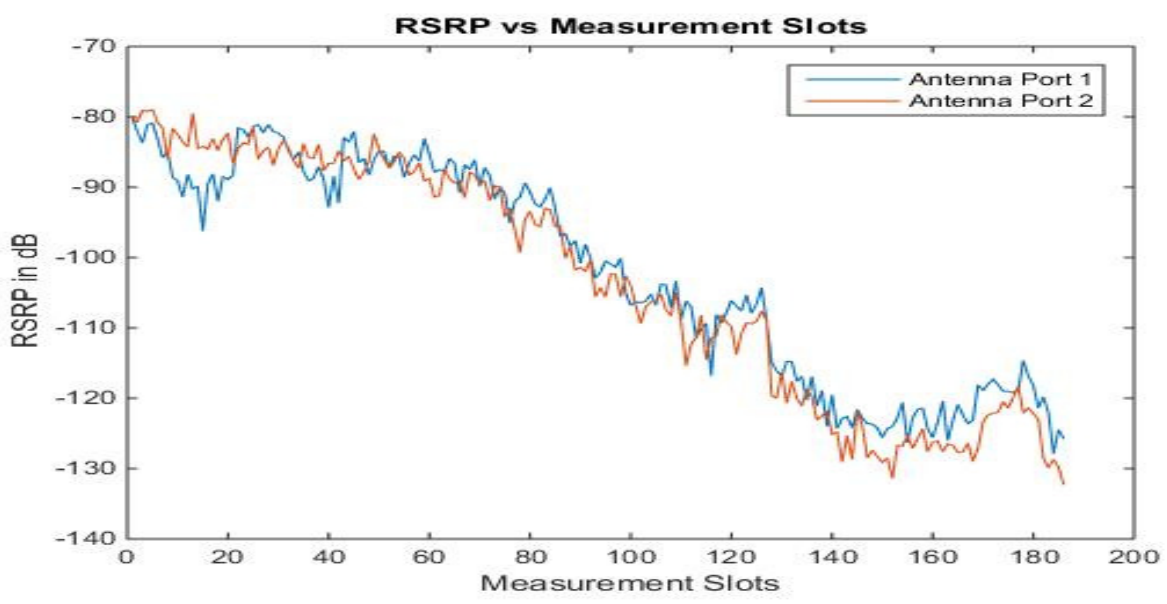

Figure 3: RSRP versus measurement slots

\subsubsection{Relationship among RSRP, RSSI and RSRQ}

A comparative analysis among RSRP, RSSI and RSRQ is reported in this subsection. Comparing the RSRP, RSRQ and RSSI graphs in Fig. 3, 4 and 5 respectively, it can be observed that both RSRP and RSSI follow the similar declining trend with increasing measurement slots. It is also observed that at a specific measurement slot RSRP is always less than RSSI. To find the possible relationship among RSRP, RSSI and RSRQ, a more elaborative study is made in which the difference between RSRP and RSSI are calculated for each measurement slot. It is noticed that lesser the difference between RSSI and RSRP, better the RSRQ. This is due to the fact that since RSRP is always less than RSSI, lesser difference between RSRP and RSSI implies lesser interference which in turn leads to better received signal quality. As an example, comparing the measurement values between measurement slot 3 and measurement slot 4, it is observed that the difference between RSRP and RSSI for port- 1 (22.8) is lesser and the RSRQ is better $(-5.8 \mathrm{~dB})$ at measurement slot 3 as compared with those for measurement slot 4 in which the difference value and RSRQ are 23.4 and $-6.4 \mathrm{~dB}$ respectively. The same conclusion could be made both for port-1 and port- 2 for all cases which is consistent with the theoretical analysis of Eq. 1.

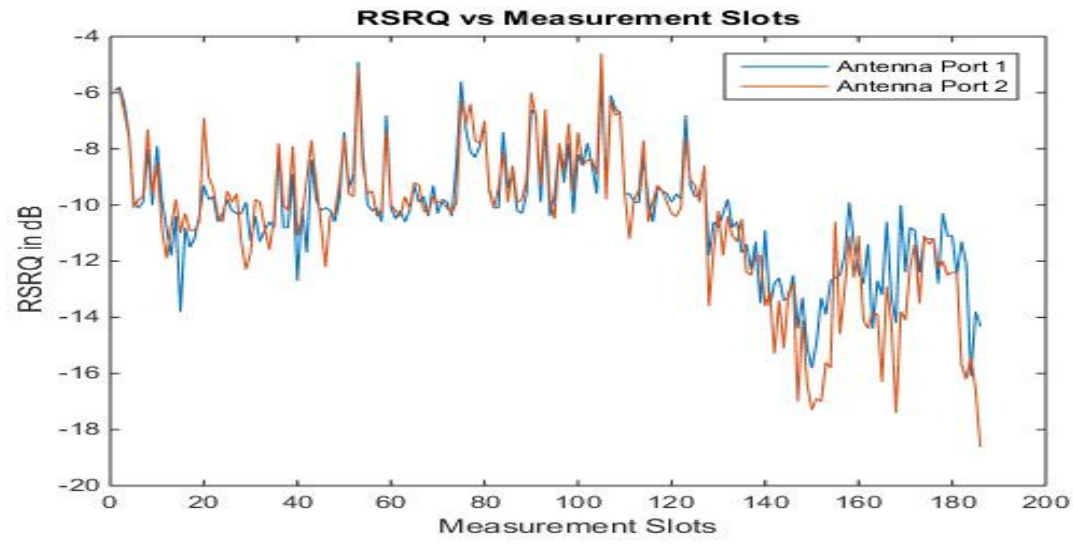

Figure 4: RSRQ versus measurement slots 


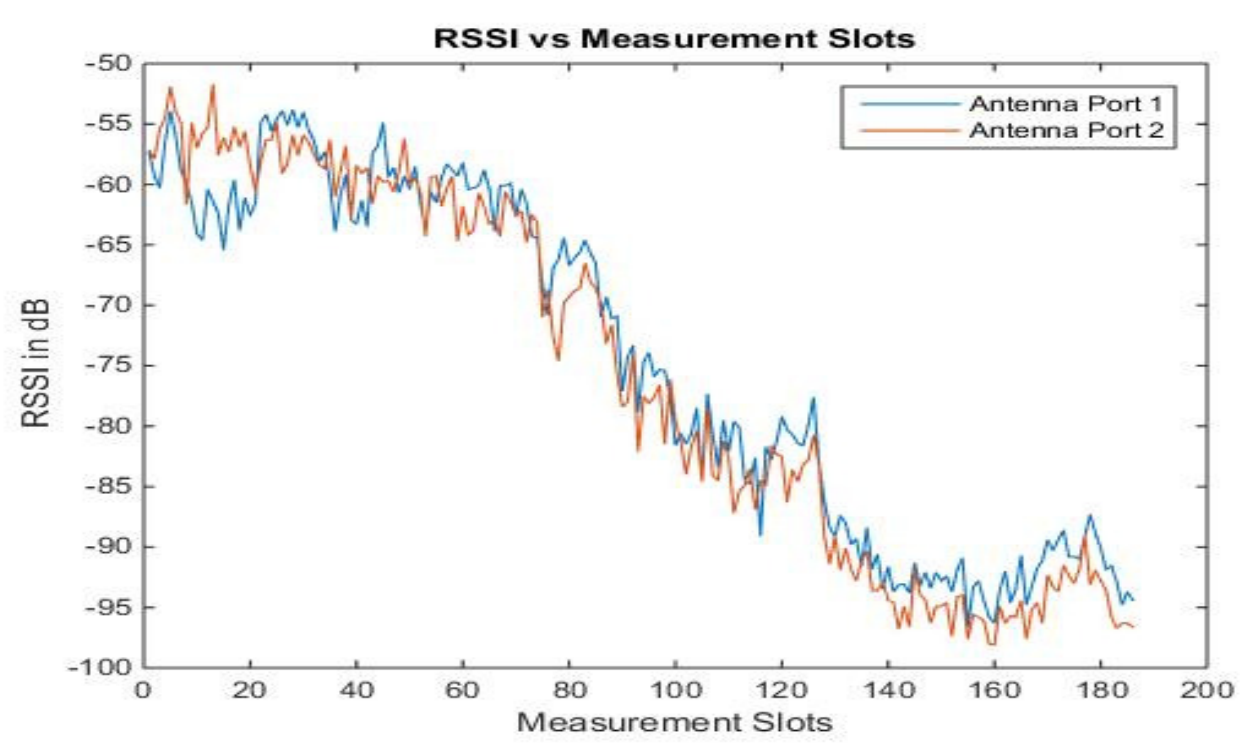

Figure 5: RSSI versus measurement slots

\subsubsection{SNR versus Throughput}

In this subsection, the effects of SNR on throughput is thoroughly studied. A comparison between the SNR vs measurement slots and throughput vs measurement slots is made as shown in Fig. 6. It is observed that the throughput is maximum (27231319 bps) at measurement slot 70 and minimum (1832937 bps) at measurement slot 146. It can also be observed that at SNR value of 5.6dB (at measurement slot 137) the throughput starts degrading lower than $10 \mathrm{Mbps}$ for more than 10 measurement slots. It is noticed that the throughput is lower than $10 \mathrm{Mbps}$ from measurement slot 137 to measurement slot 178 .

To study the possible relationship between SNR and PDSCH (Physical Downlink Shared Channel) throughput, the average amount of allocated PRBs has been calculated and found to be 32 PRBs approximately and then the throughput is computed for each measurement slot for respective maximum SNR using the following equation.

$$
\mathrm{C}=\beta . \mathrm{W} \cdot \log _{2}(1+\alpha \cdot \mathrm{snr})
$$

Where, $\mathrm{C}$ is the throughput in bps, $\mathrm{W}$ is the bandwidth of one $\mathrm{RB}$ (i.e. $180 \mathrm{KHz}$ ), snr is the signal to noise ratio expressed as a linear power ratio, and the typical value of $\alpha$ is between 0.1 to 1 .

The graphical representation of the maximum SNR versus corresponding throughput computed using Eq. 2 is shown in Fig. 7. It is seen that if the SNR is good for a measurement slot, higher throughput is achieved which is consistent with link adaptation theory.

Furthermore, the PRB percentage and modulation percentage graphs for $\mathrm{t}_{\max }=70$ at which peak throughput occurs and $t_{\min }=146$ at which lowest throughput occurs are depicted in Fig. 8 and Fig. 9. Comparing Fig. 8 and Fig. 9, it is seen that for measurement slot, $t_{\max }=70$ at which maximum throughput occurs, the percentage of 64QAM (6 bits/symbol) modulation scheme is higher than other two lower order modulation schemes, whereas for measurement slot, $\mathrm{t}_{\min }=146$ at which lowest throughput occurs, the percentage of QPSK (2 bits/symbol) modulation scheme dominates, and higher order modulation scheme such as 16QAM or 64QAM has not been selected for signal transmission at all. It is also observed that at measurement slot, $\mathrm{t}_{\max }=70$, the 
International Journal of Wireless \& Mobile Networks (IJWMN) Vol. 7, No. 4, August 2015

probability of allocating 50 PRBs to the UEs by the eNB is highest (73.4\%), whereas at $\mathrm{t}_{\min }=146$, the probability of allocating 0 PRB is highest $(52 \%)$. It can be predicted that at $\mathrm{t}_{\max }$, more PRBs are allocated to the UE by eNB due to the good channel condition (i.e. good CQI report or good SNR). The higher value of SNR causes higher order modulation scheme (64QAM) to be selected for data transmission which implies more bits per symbol are transmitted and thus higher throughput is achieved at $t_{\max }$. At measurement slot, $t_{\min }$, the channel condition seems to be worse i.e. SNR is poor and that's why lower order modulation scheme (QPSK) is selected as well as the probability of allocating more PRBs is decreased. As fewer number of bits per symbol are transmitted in QPSK, the achieved throughput is lower at $t_{\min }$. This process of selecting MCS based on channel condition is called link adaptation in LTE.

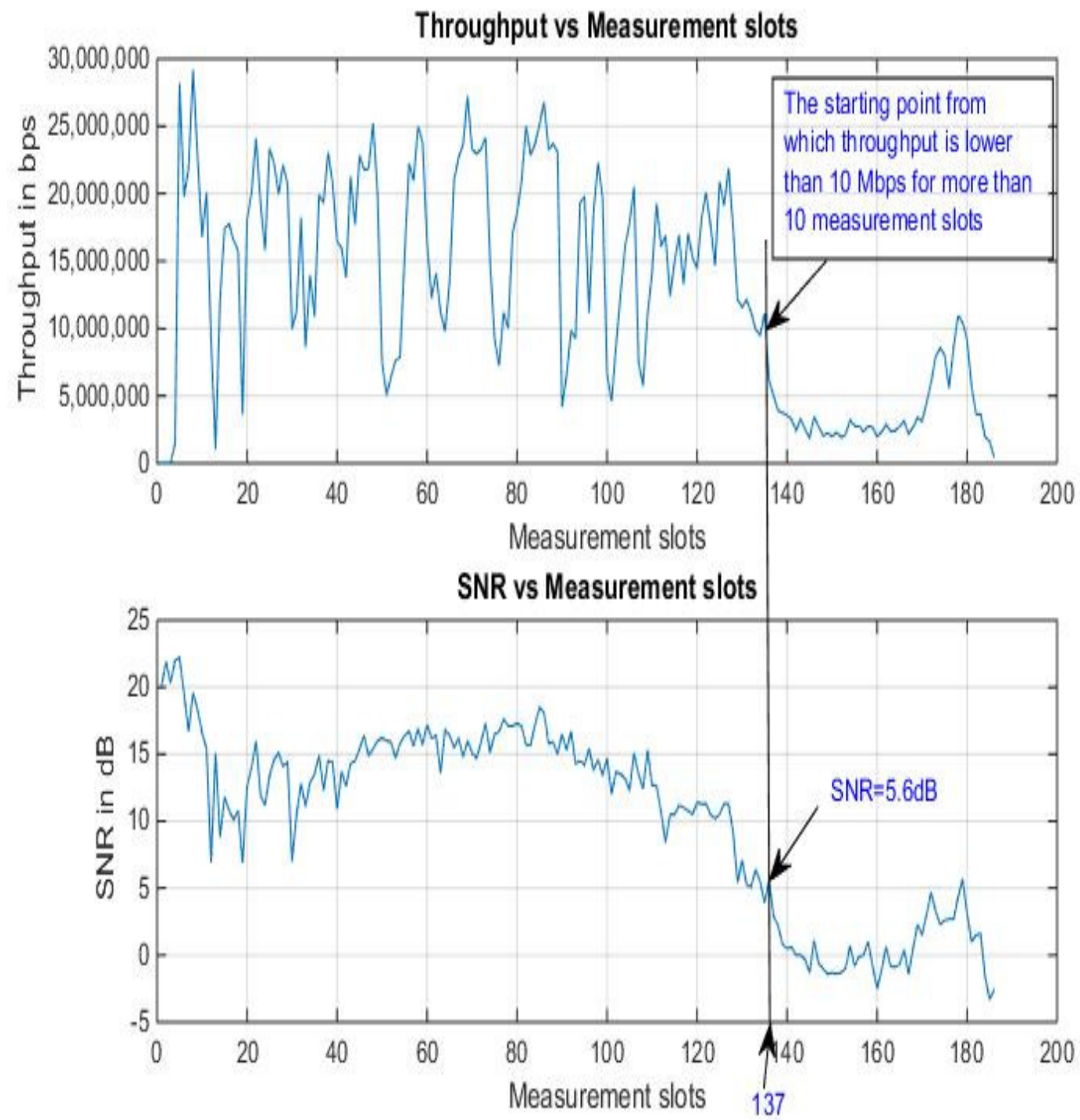

Figure 6: Finding SNR at which the throughput starts to degrade (i.e. when the throughput is lower than 10 Mbps for more than 10 measurement slots) 
International Journal of Wireless \& Mobile Networks (IJWMN) Vol. 7, No. 4, August 2015

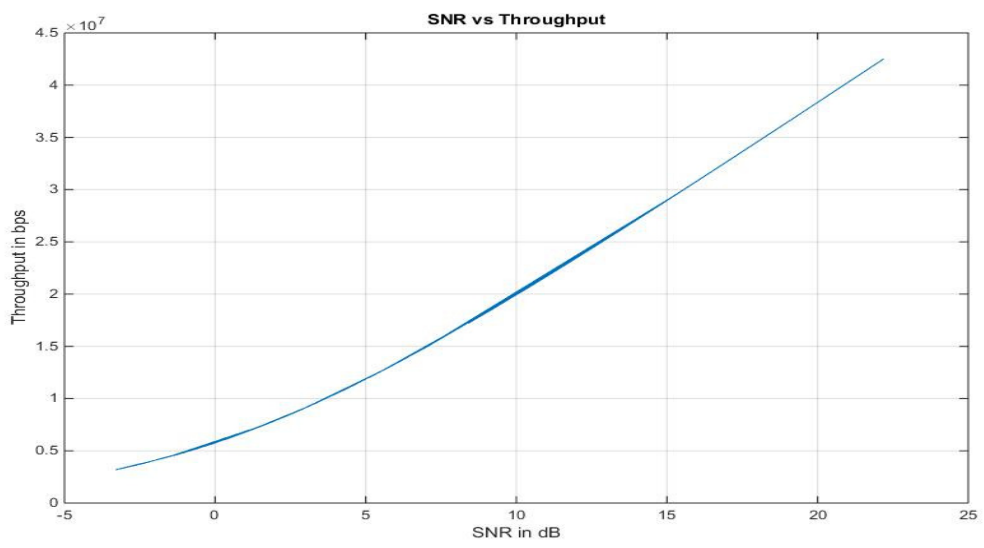

Figure 7: SNR versus throughput
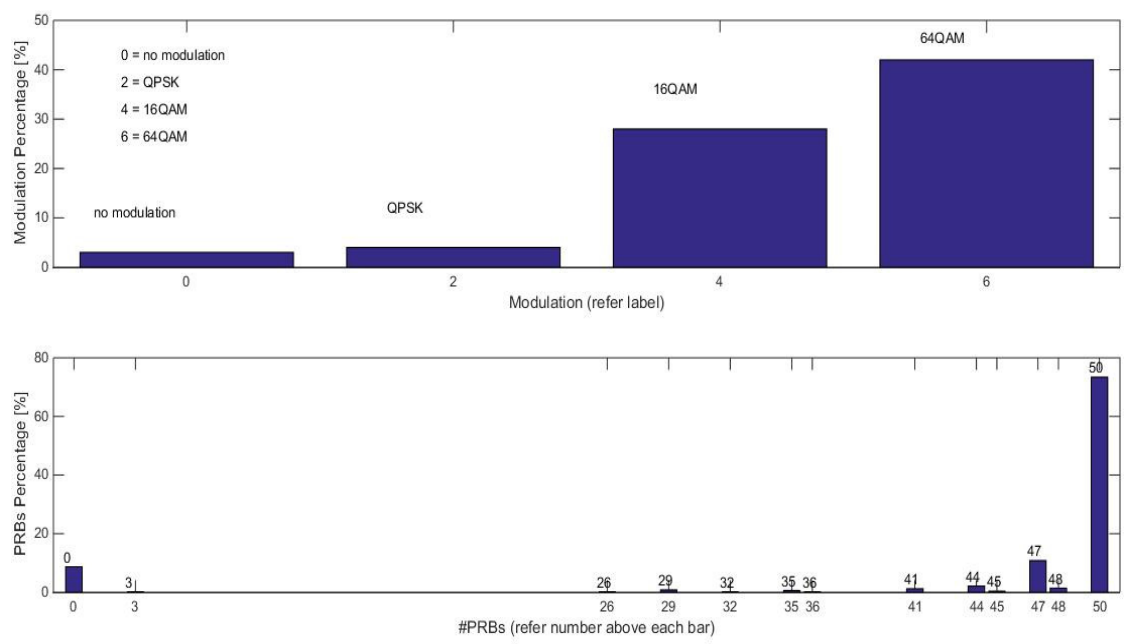

Figure 8: Modulation percentage and PRB percentage at measurement slot, $\mathrm{t}_{\max }=70$ at which maximum throughput occurs
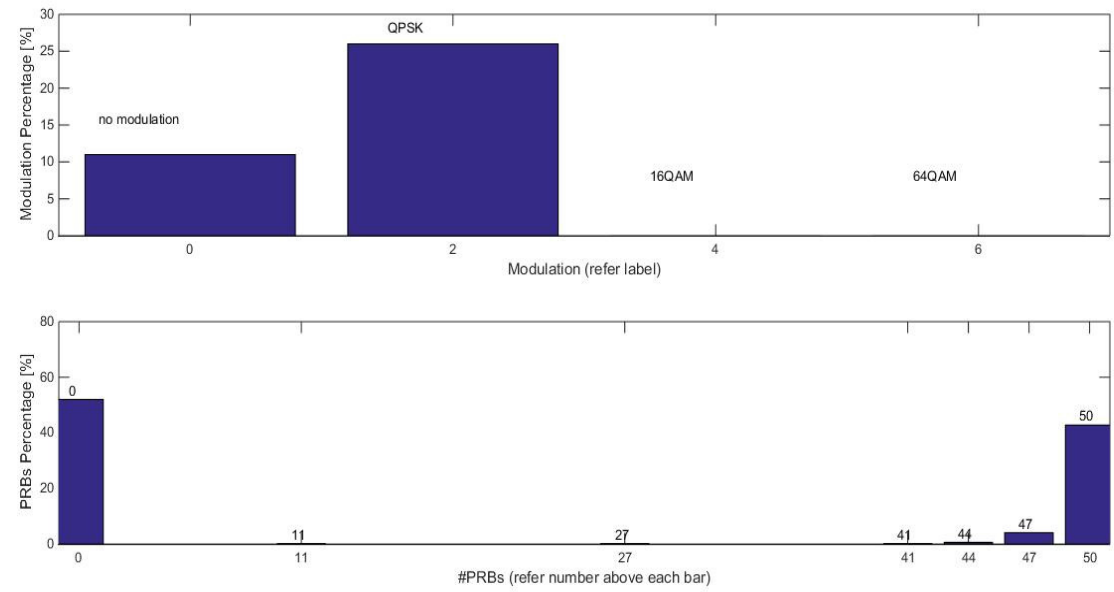

Figure 9: Modulation percentage and PRB percentage at measurement slot, $\mathrm{t}_{\min }=146$ at which lowest throughput occurs 
International Journal of Wireless \& Mobile Networks (IJWMN) Vol. 7, No. 4, August 2015

\subsection{Case 2: Study of intra-eUTRAN Handover Events}

In Case 2, the intra-eUTRAN handover test was performed using NEMO Handy in a handover scenario where total three FTP download sessions were run downloading the file of 100MB while the UE was travelling on a train from Redfern to Petersham. Similar to Case 1, the network with which the test was performed was an LTE FDD network with a bandwidth of 10MHz. Total 13 cells served the UE during the test period, with 13 successful handover events occurred within LTE eUTRAN at $100 \%$ HO success rate. In this subsection, the intra-eUTRAN handover events occurred during the test are analysed.

A graphical analysis of RSRP and RSRQ of the serving cells during the test period is made using Fig. 10. Since RSRP and RSRQ values are negative, the closer the value to 0 , the better is the RSRP/RSRQ. Hence, the maximum and minimum RSRP values are found to be $-66.1 \mathrm{dBm}$ and $125 \mathrm{dBm}$ respectively, whereas the maximum and minimum RSRQ values are seen to be $-1.9 \mathrm{~dB}$ and $-15 \mathrm{~dB}$ respectively. The average RSRP and RSRQ values are calculated and found as $92.8435 \mathrm{dBm}$ and $-7.86949 \mathrm{~dB}$ respectively which is an indication of average quality LTE FDD network.

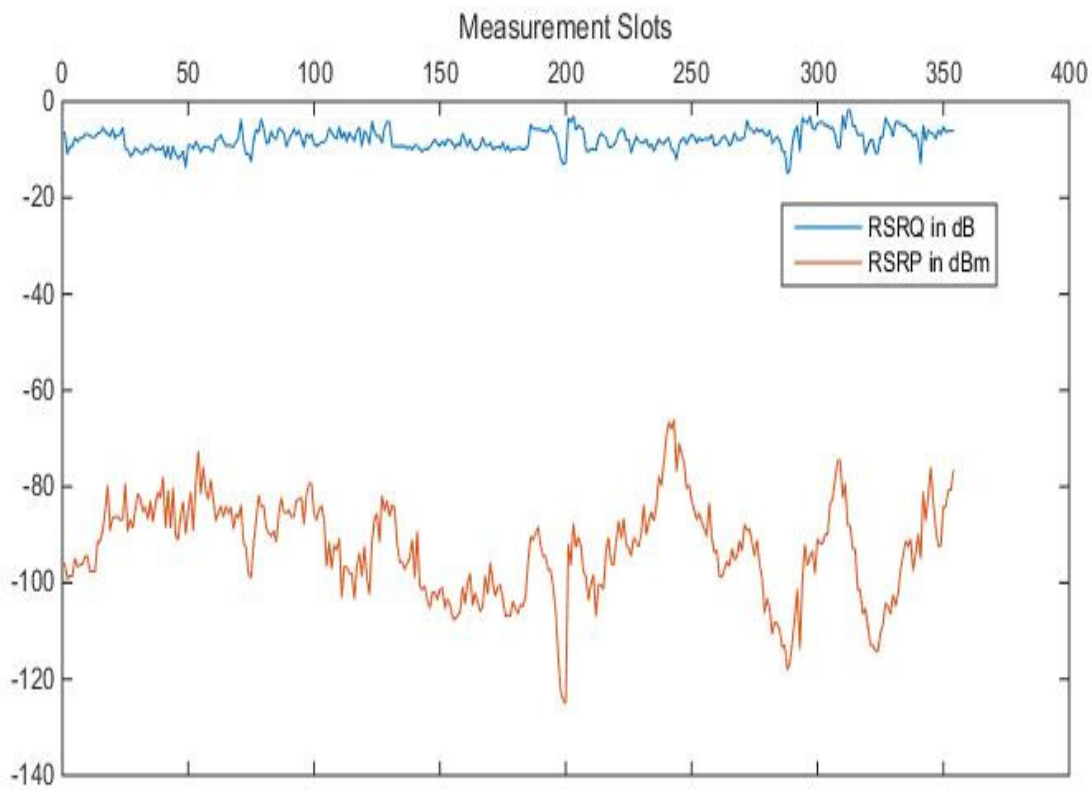

Figure 10: RSRP and RSRQ plot of the serving cells

A pictorial representation of RSRP and RSRQ for one successful handover event is depicted in Fig. 11. In this figure, the handover event made from PCI (Physical Cell Identity) 210 to PCI 212 is considered and the corresponding RSRP and RSRQ graph for the old cell (i.e. PCI 210) and the new cell (i.e. PCI 212) is shown. From Fig. 11, it is seen that the HO has occurred at slot 50, and the HO process triggers exactly at time 32:03.1. This successful HO was made with delay 0.044s. As seen from the figure, the HO takes place due to the fact that both the RSRP and RSRQ of the neighbor cell 212 exceed compared with those of the serving cell 210 for certain period of time. At slot 50, the RSRP values of PCI 210 and PCI 212 are $-89.9 \mathrm{dBm}$ and $-81.1 \mathrm{dBm}$ respectively, and the RSRQ values of PCI 210 and PCI 212 are $-13.7 \mathrm{~dB}$ and $-9.2 \mathrm{~dB}$ respectively, implying that the RSRP/RSRQ of PCI 210 drops below the RSRP/RSRQ values of PCI 212. Consequently, handover occurs from cell 210 to cell 212 to maintain the quality of ongoing data session. 


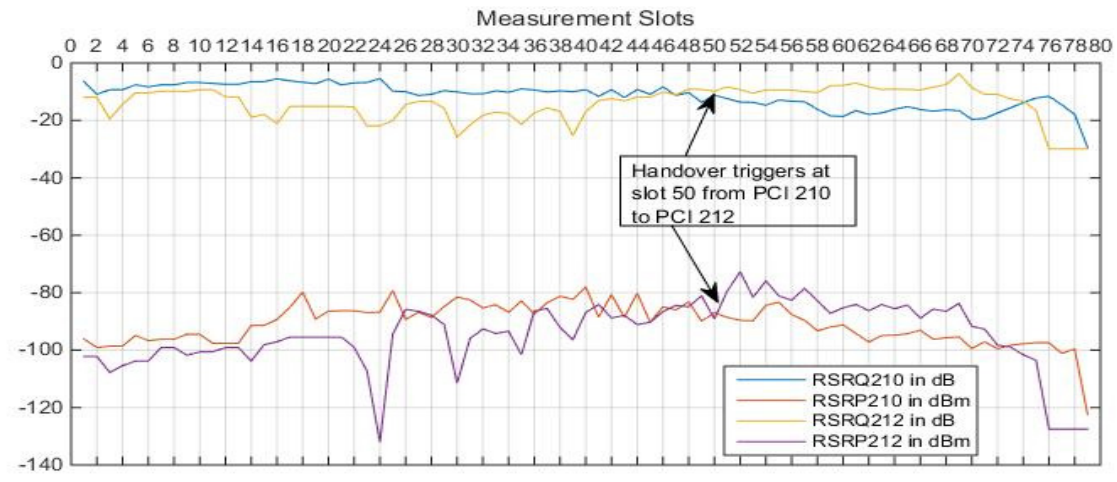

Figure 11: RSRP and RSRQ plot for one successful handover from PCI 210 to PCI 212

A repeated back and forth $\mathrm{HO}$ event between two base stations (eNodeBs) namely ping-pong $\mathrm{HO}$ is also observed and shown in Fig. 12. It is noticed that during the test, ping pong handover was encountered between PCI 210 and PCI 212. The ping-pong HO might happen due to the frequent movement of the terminal between the source and the target eNodeB, passing trees, buildings or other obstacles, or due to the high fluctuation of the signal at the overlapped boundary of the eNodeBs. The location area of users, their movement and speed as well as the coverage parameters are the key considerations that can lead ping-pong HO.

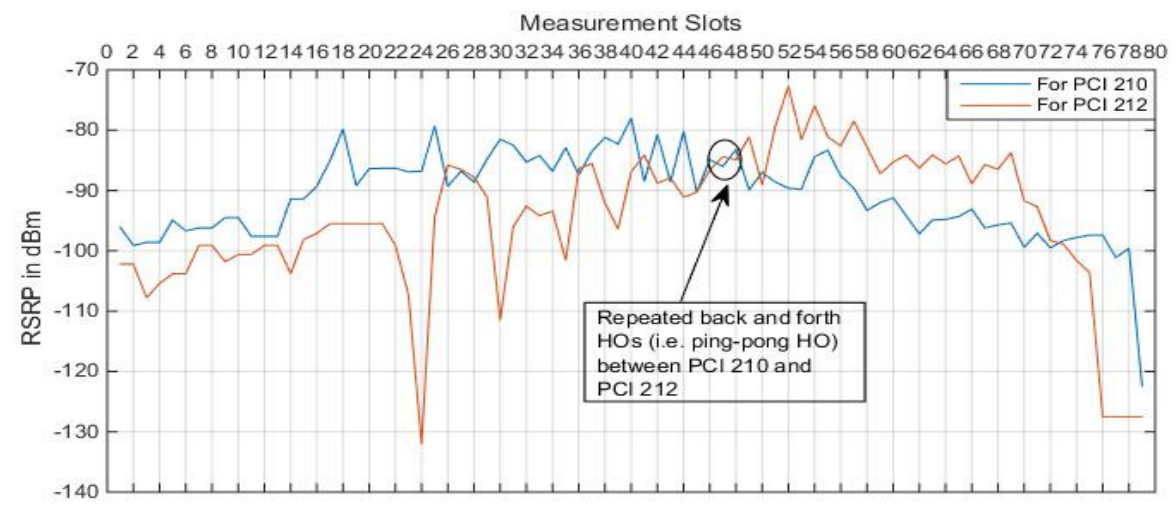

Figure 12: RSRP plot to show ping-pong handover

\section{CONCLUSION}

In this work, an analysis of some practical measurement results recorded from a live LTE FDD network of Australia are presented to verify the possible relationships among LTE measurements such as SINR, RSRP, RSSI and RSRQ as well as to evaluate the effects of SNR on throughput. Furthermore, the handover events occurred within LTE-eUTRAN during the test period are studied. It is observed that RSRP and SNR are proportional to each other on average, and lesser the difference between RSSI and RSRP, better is the RSRQ, and if the SINR is better for a measurement slot, higher throughput is achieved. It is also noticed that when the RSRP and/or RSRQ of a serving cell drops below the RSRP/RSRQ of neighbor cell, the handover event occurs to maintain the ongoing call or data session. All of these observations are found to be consistent with theory. Our future work includes the performance analysis of a live LTE TDD network based on practical measurement results. 
International Journal of Wireless \& Mobile Networks (IJWMN) Vol. 7, No. 4, August 2015

\section{REFERENCES}

[1] A. Ghosh and R. Ratasuk (2011), Essentials of LTE and LTE-A, Cambridge UK, New York: Cambridge University Press.

[2] F. Afroz, K. Sandrasegaran and P. Ghoshal (2014), "Performance Analysis of PF, M-LWDF and EXP/PF Packet Scheduling Algorithms in 3GPP LTE Downlink," Australasian Telecommunication Networks and Applications Conference (ATNAC), November 2014, pp. 87-92.

[3] F. Afroz (2014), Research in 4G Mobile Network, MES Project, University of Technology, Sydney.

[4] J. Bannister, P. Mather and S. Coope (2004), Convergence Technologies for 3G Networks: IP, UMTS, EGPRS and ATM, John Wiley \& Sons.

[5] G. Piro, L. A. Grieco, G. Boggia, F. Capozzi, and P. Camarda (2011), "Simulating LTE cellular systems: an open- source framework," IEEE Transactions on Vehicular Technology, vol. 60, pp. 498513.

[6] H. Holma and A. Toskala (2009), LTE for UMTS: OFDMA and SC-FDMA based radio access, John Wiley \& Sons Ltd.

[7] L. Luan, M. Wu, J. Shen, J. Ye and X. He (2012), "Optimization of Handover Algorithms in LTE High-speed Railway networks," International Journal of Digital Content Technology and its Applications (JDCTA), vol. 6, no. 5.

[8] D. G. Ainscough (2001), The Evolution and Future of Mobile Communication Systems, ICT Report. Available: http://www.foresightfordevelopment.org

[9] H. Holma and A. Toskala (2011), LTE for UMTS: Evolution to LTE-Advanced, Chichester, West Sussex: John Wiley \& Sons.

[10] S. Sesia, I. Toufik, and M. Baker (2009), LTE- The UMTS Long Term Evolution: From Theory to Practice, Chichester, U.K. Wiley.

[11] 3GPP TS 36.214 version 11.1.0 Release 11 (2013), "LTE; Evolved Universal Terrestrial Radio Access (E-UTRA); Physical layer; Measurements”. Available: http://www.etsi.org 